\section{Klinische Bedeutung des Helicobacter pylori: Erreger oder Saprophyt?}

Frage: Gibt es Untersuchungen von Magenschleimhautbiopsien bei Helicobacterpositiven Menschen ohne Magenbeschwerden? Mir scheint die Frage „Helicobacter: Erreger oder Saprophyt?“ nicht ausreichend geklärt.

Antwort: Seit über 100 Jahren gilt das Dogma "keine Säure kein Ulkus», wobei ein Ungleichgewicht zwischen schleimhaut-aggressiven und -protektiven Faktoren die entscheidende pathogenetische Rolle für die Ulkusentstehung spielt. Dieses Dogma war der Grundstein für das Studium der Magenphysiologie und -pathophysiologie. Darauf gründen sich die Therapieoptionen.

1984 wurde von den beiden australischen Ärzten Warren und Marschall (17) die Hypothese aufgestellt, daß ein im Magen nachgewiesenes Bakterium, Helicobacter pylori (HP), ätiologisch eine Rolle für die Entwicklung von Gastritis und gastroduodenalen Ulzera spiele. Ihre Hypothese wurde durch epidemiologische Studien unterstützt, die zeigten, daß bei bis zu 90\% der Patienten mit einem Ulcus duodeni und $70 \%$ der Patienten mit einem Ulcus ventriculi Helicobacter pylori nachgewiesen werden kann. Schließlich haben Interventionstudien Beweise zur pathogenetischen Bedeutung des Helicobacter pylori bei der Ulkusentstehung erbracht, da nach einer Eradikationstherapie die 5-Jahres-Ulkusrezidivrate auf unter $10 \%$ reduziert werden kann, ähnlich wie nach einer Magenteilresektion oder einer trunkalen Vagotomie. Aufgrund dieser Daten wurde von den gastroenterologischen Fachgesellschaften die Empfehlung zur Eradikationstherapie bei der Behandlung des peptischen Ulkus ausgesprochen $(3,12)$.

Dennoch sind die genauen Pathomechanismen der Helicobacter-induzierten gastroduodenalen Läsionen trotz intensiver Forschungen auf diesem Gebiet noch nicht gänzlich bekannt. Die Gründe dafür sind vielfältig, vor allem handelt es sich bei der HP-Besiedlung des Magens um ein sehr komplexes Geschehen mit zahlreichen Facetten $(1,2,9,24)$ :

1. Der Zeitraum zwischen Infektion und klinischer Manifestation kann bis zu mehrere Dekaden betragen. Die Infektion findet sich überwiegend im frühen Kindesalter statt, die klinische Manifestation erst im Erwachsenenalter; das heißt, es handelt sich hierbei um einen langsamen Prozess, bei dem weitere Faktoren eine Rolle spielen müssen.

2. Nicht alle Helicobacter-positiven Personen sind krank. Die HP-Prävalenz liegt in den Entwicklungsländern bei über 90\% und in den Industrieländern altersabhängig zwischen 20 und 60\%. Die große Mehrheit dieser Patienten bleibt lebenslang asymptomatisch.

3. Die individuelle klinische Manifestation ist sehr variabel. Zu den Helicobacter-assoziierten gastroduodenalen $\mathrm{Pa}$ thologien zählen die Gastritis, das Ulcus ventriculi, das Ulcus duodeni und eventuell auch das Magenkarzinom und das primäre Magenlymphom (MALT-Lymphom) (7, 23). Die Erkrankungen haben zum Teil gegenteilige Auswirkungen, zum Beispiel haben Patienten mit einem Ulcus duodeni ein deutlich niedrigeres Risiko, an einem Magenkarzinom zu erkranken, als Patienten mit einem Ulcus ventriculi oder die normale Bevölkerung.

Die Helicobacterbesiedlung des Magens führt zu einer Pangastritis mit vorwiegender Kolonisation der nicht Säure bildenden Regionen wie Antrum oder Kardia $(11,20)$. Histologische Untersuchungen der Magenschleimhaut der verschiedenen Regionen bei Patienten zeigen uneinheitliche Verteilungsmuster bzw. Gastritistypen $(1,2,9,24)$ :

- Patienten mit einem Ulcus duodeni bzw. einem pylorusnahen Ulcus ventriculi haben vorwiegend eine antrumbetonte chronisch aktive Gastritis.

- Patienten mit einem proximalen Ulcus ventriculi haben vorwiegend eine korpusbetonte chronisch aktive Gastritis. Ein Teil dieser Patienten kann das Bild einer multifokalen atrophischen Gastritis mit intestinaler Metaplasie aufweisen, welches häufig im Zusammenhang mit einem Magenkarzinom beobachtet wird.

- Asymptomatische Patienten weisen in bis zu 30\% eine normale Mukosa auf, insbesondere Kinder und Jugendliche, oder lediglich eine oberflächliche Pangastritis mit wenig Entzündungsaktivität und Umbauprozessen $(7,16,19$,
22). Daten diesbezüglich sind begrenzt, denn eingehende endoskopisch-bioptische Untersuchungen dieser Patientengruppen sind aus ethischen Gründen kaum möglich.

Diese Befunde deuten darauf hin, daß für die klinischen Manifestationen der HPInfektion patientenbezogene Determinanten eine Rolle spielen.

Der sekretorische Status bzw. die Säureproduktion könnte eine wichtige Rolle für die klinische Manifestation bzw. für die Bildung eines bestimmten Gastritistypes spielen $(4,9,15)$. Maßnahmen zur effektiven Säureproduktionshemmung, sei es medikamentös mit $\mathrm{H}_{2}$-Blockern oder insbesondere mit Protonenpumpeninhibitoren, oder chirurgisch durch Vagotomie, führen zu einem Rückgang der HP-Kolonisation und zum Teil zum Rückgang der Gastritisaktivitäten im Antrum, während die Entzündungsparameter im Korpus zunehmen. Es wird diskutiert, daß ein hyperazider Magen zu einer antrumbetonten Gastritis führt, während es bei einem hypoaziden Magen häufig zur Bildung einer korpusbetonten Gastritis kommt. Genetische Prädispositionen, Ernährungsstatus sowie individuelle Risikofaktoren wie Nikotinabusus oder Streß werden als mögliche Determinanten für den sekretorischen Status diskutiert, welche die Masse der säureproduzierenden Parietalzellen bestimmen und somit pathogenetisch an der Ulkusbildung beteiligt sind $(2,9)$. Auf der anderen Seite führt eine helicobacter-induzierte antrumbetonte Gastritis über unterschiedliche Mechanismen zur Hypergastrinämie und konsekutiv zur Erhöhung der basalen Säureproduktion (BAO) sowie zur postprandialen anhaltenden und überschießenden maximalen Säureproduktion (MAO und PAO) (9). In der Ulkuspathogenese können Säure und Helicobactergastritis sich somit gegenseitig positiv beeinflussen.

Helicobacter pylori ist auch in der Lage, eine Reihe lokaler Schädigungen an der Magenschleimhaut hervorzurufen (2, 9, 14, 24):

1. Die Geißel des Bakteriums ermöglicht es ihm, die Mukusschicht durchzudringen und somit diesen Säureabwehrmechanismus zu durchbrechen.

2. Bestimmte Helicobacter-Stämme sind in der Lage, Toxine bzw. für die Magenschleimhaut toxische Genprodukte zu bilden, und somit Schleimhaut di- 
rekt zu schädigen. Hierbei zu erwähnen sind die Pathogenitätsfaktoren CagA und VacA.

3. Die Produktion von TransformingGrowth-Factor-Beta, einem wichtigen Mediator in der Wundheilung, kann bei der HP-Gastritis vermindert sein.

4. Die chronische Infektion kann zur lokalen Produktion von Zytokinen, vor allem der proinflammatorischen Interleukine TNF, IL2 und IL8, mit konsekutiver Aktivierung von Granulozyten und Lymphozyten führen. Eine solche ständige Aktivierung des lokalen Immunsystems im Rahmen einer chronischen Entzündung wird derzeit als ein Pathomechanismus für die Malignomentwicklung bei der HP-Infektion diskutiert.

Zusammenfassend kann der Helicobacter pylori bei prädisponierten Patienten bzw. bei Vorhandensein von Risikofaktoren sowohl durch Verstärkung der Säureproduktion als auch durch direkte Schleimhautschädigungen die Ulkusbildung fördern. Er spielt daher eine zentrale Rolle in der Ulkuspathogenese. Die Pathomechanismen lassen sich in das Konzept des Ungleichgewichtes zwischen schleimhaut-aggressiven und -protektiven Faktoren integrieren.

Dennoch ist unklar, welche Bedeutung der Helicobacter bei der überwiegenden Mehrheit der HP-Träger, insbesondere bei den asymptomatischen Patienten hat. Aufgrund der sehr hohen Prävalenz der Infektion sowie der ausgezeichnete Anpassung des Keimes an den Wirt wird die Möglichkeit einer physiologischen Funktion dieser Besiedlung des Magens durch den Helicobacter diskutiert. Dafür gibt es derzeit folgende Hinweise:

1. Da die HP-Infektion in den Entwicklungsländern mit geringem hygienischem Standard sehr verbreitet ist, ist es möglich, daß durch die Induktion einer Hyperazidität im Magen der unspezifische Abwehrmechanismus des Magens gegenüber anderen humanpathogenen Keimen verstärkt wird $(1,2)$.

2. Anhand der Untersuchungen des Helicobacterlysates und dessen Genom wurde diskutiert, daß unter den Stoffwechselprodukten von Helicobacter bakterizide Peptide sind (21), welche die unspezifischen lokalen Immunabwehrmechanismen des Magens unterstützen.

3. Es wurde festgestellt, daß eine orale Immunisierung mit Cholerabakterien zu einer bis zu 80 -fach höheren loka- len IgA-Produktion bei HP-Trägern als bei HP-negativen Patienten führt (18). Die lokale spezifische Immunantwort des Magen könnte damit verstärkt werden.

4. Untersuchungen an asymptomatischen HP-Trägern zeigten, daß im Gegensatz zu Ulkuspatienten eine Eradikation zu einer verstärkten pentagastrininduzierten Säuresekretion führt (6). Dieser Befund würde die klinischen Beobachtungen erklären, daß nach einer Eradikationstherapie Inzidenz und Schwere einer Refluxösophagitis zunehmen können (13). Eine HP-Besiedlung scheint auch die Säurehemmerwirkung von Protonenpumpeninhibitoren wie Omeprazol zu potenzieren (10). Der Helicobacter scheint bei Patienten mit einer korpusbetonnten Gastritis einen protektiven Effekt auf die Refluxkrankheit auszuüben (5).

Somit scheint eine HP-Besiedlung des Magens möglicherweise auch für den Wirt eine physiologische Funktion zu haben.

Aufgrund der vorliegenden Daten muß man die HP-Infektion des Magens sehr differenziert betrachten. Die Ulkuspathogenese gilt als gesichert. Es sind noch viele Fragen offen, insbesondere hinsichtlich der Malignomentwicklung, ein multifaktorielles Geschehen. Ein HP-Nachweis ist jedoch nicht gleichbedeutend mit einer gastroduodenalen Krankheit. Endoskopisch-histopathologische Untersuchungen sind erforderlich, um den Infektionstatus genau festzustellen. Daraus ergeben sich die Therapieindikationen gemäß den Empfehlungen der Fachgesellschaften (3, 12). Da eine physiologische Funktion der Helicobacterbesiedlung derzeit noch nicht ausgeschlossen werden kann, ist eine generelle Eradikation bei allen Helicobacterträgern, insbesondere bei asymptomastichen Patienten, zum Beispiel um eine mögliche Karzinomentwicklung bei einem verhältnismäßig geringen Patientenanteil zu verhindern, derzeit kritisch zu betrachten; hierzu ist eine genaue Risiko-Nutzen-Stratefizierung erforderlich (1).

\section{Literatur}

1 Blaser, M. J.: Not all Helicobacter pylori strains are created equal: should all be eliminated? Lancet 349 (1997), 1020-1022

2 Blaser, M. J.: Helicobacter pylori and gastric diseases. BMJ 316 (1998), 1507-1510.

3 Caspary, W. F, R. Arnold, E. Bayerdörfer et al. Diagnostik und Therapie der Helicobacter-py-
lori-Infektion. DGVS-Leitlinien 1. Z. Gastroenterol. 34 (1996), 392-401.

4 Danon, S. J., J. L. O'Rourke, N. D. Moss, A. Lee: The importance of local acid production in the distribution of Helicobacter felis in the mouse stomach Gastroenterology 108

5 El-Serag, H. B. A. Sonnenberg . M. Jamal et al.: Corpus gastritis is protective against reflux oesophagitis. Gut 45 (1999), 181-185.

6 Feldman, M., B. Cryer, E. Lee: Effects of Helicobacter pylori gastritis on gastric secretion in healthy human beings. Am. J. Physiol. 274 (1998), G1011-1017.

7 Fischbach, W.: MALT-Lymphome des Magens. Dtsch med Wschr 124 (1999), 1142-1147.

8 Gottrand, F., F. Cullu, D. Turck et al.: Normal gastric histology in Helicobacter pylori-infected children. J. Pediatr. Gastroenterol. Nutr. 25 (1997), 74-78.

9 Graham, D. Y.: Helicobacter pylori infection in the pathogenesis of duodenal ulcer and gastric cancer: a model. Gastroenterology 113 (1997), 1983-1991.

10 Gillen, D., A. A. Wirz, W. D. Neithercut, J. E. Ardill, K. McColl: Helicobacter pylori infection potentiates the inhibition of gastric acid secretion by omeprazole. Gut 44 (1999), 468-475.

11 Holtmann, G., H. Goebell: Aktuelle Diagnostik der Dypepsie. Dtsch med Wschr 123 (1998), 461-465.

12 Howden, C. W.: Guidelines for the management of Helicobacter pylori infection. Ad Hoc Committee on Practice Parameters of the American College of Gastroenterology. Am. J. Gastroenterol. 93 (1998), 2330-2338.

13 Labenz, J., A. L. Blum, E. Bayerdorffer, A. Meining, M. Stolte, G. Borsch: Curing Helicobacter pylori infection in patients with duodenal ulcer may provoke reflux esophagitis. Gastroenterology 112 (1997), 1442-1447.

14 Lehmann, F. S., G. A. Stalder: Hypotheses on the role of cytokines in peptic ulcer disease. Eur. J. Clin. Invest. 28 (1998), 511-519.

15 Lee, A., M. F. Dixon, S. J. Danon et al.: Local acid production and Helicobacter pylori. Eur. J. Gastroenterol. Hepatol. 7 (1995), 461-465.

16 Loffeld, R. J., E. Stobberingh, J. A. Flendrig, J. W. Arends: Presence of Helicobacter pylori in patients with non-ulcer dyspepsia revealing normal antral histological characteristics. Digestion 47 (1990), 29-34

17 Marshall, B. J., J. R. Warren: Unidentified curved bacilli in the stomach of patients with gastritis and peptic ulceration. Lancet 1 (1984), 1311-1315.

18 Mattsson, A., H. Lonroth, M. Quiding-Jarbrink A. M. Svennerholm: Induction of B cell responses in the stomach of Helicobacter pylori- infected subjects after oral cholera vaccination. J Clin. Invest. 102 (1998), 51-56.

19 Misra, V., S. P. Misra, M. Dwivedi, P. A. Singh: Point prevalence of peptic ulcer and gastric histology in healthy Indians with Helicobacter pylori infection. Am. J. Gastroenterol 92 (1997), 1487-1491.

20 Nordmann, A., O. Friesewinkel, U. Scheurer, K. Gyr: Dyspepsie im Alter. Dtsch med Wschr 123 (1998), 557-580.

21 Putsep, K., C. I. Branden, H. G. Boman, S. Normark: Antibacterial peptide from $\mathrm{H}$. pylori. Nature 398 (1999), 671-672.

22 Prabhu, S. R., S. Ranganathan, D. N. Amarapurkar: Helicobacter pylori in normal gastric mucosa. J. Assoc. Physicians. India 42 (1994), 863.

23 Seelis, R.E.A., W. Dohmen.: Kurzzeiteradikation der Helicobacter-pylori-Infektion. Dtsch med Wschr 123 (1998), 103-108.

24 Smoot, D. T.: How does Helicobacter pylori cause mucosal damage? Gastroenterology 113 (6 Suppl) (1997), S31-S34.

PD Dr. med. Huan N. Nguyen

Medizinische Klinik III

Universitätsklinikum der RWTH

Pauwelstraße 30

52074 Aachen

hnguyen@post.klinikum.rwth-aachen.de 